\title{
Body weight changes and incidence of cachexia after stroke
}

\author{
Nadja Scherbakov ${ }^{1,2,3 *}$, Charlotte Pietrock ${ }^{3}$, Anja Sandek $^{4,5}$, Nicole Ebner ${ }^{4,5}$, Miroslava Valentova $^{4,5}$, \\ Jochen Springer ${ }^{1,6}$, Joerg C. Schefold ${ }^{7}$, Stephan von Haehling ${ }^{4,6}$, Stefan D. Anker ${ }^{1,6,8}$, Kristina Norman ${ }^{9,10}$, \\ Karl Georg Haeusler ${ }^{3} \&$ Wolfram Doehner ${ }^{1,2,3}$ \\ ${ }^{1}$ BIH Center for Regenerative Therapies (BCRT), Charité - Universitätsmedizin Berlin, Germany, ${ }^{2}$ Department of Cardiology (Virchow Klinikum), Charité Universitätsmedizin \\ Berlin, and German Center for Cardiovascular Research (DZHK), partner site Berlin, Germany, ${ }^{3}$ Center for Stroke Research Berlin (CSB), Charité - Universitätsmedizin Berlin, \\ Germany, ${ }^{4}$ Department of Cardiology and Pneumology, University Medicine Goettingen (UMG), Goettingen, Germany, ${ }^{5}$ German Centre for Cardiovascular Research (DZHK), \\ partner site Goettingen, Goettingen, Germany, ${ }^{6}$ Division of Cardiology and Metabolism, Department of Cardiology (CVK), Charité, Berlin, Germany, ${ }^{7}$ Department of Intensive \\ Care Medicine, Inselspital, Bern University Hospital, Switzerland, ${ }^{8}$ German Centre for Cardiovascular Research (DZHK), partner site Berlin, Berlin, Germany, ${ }^{9}$ Research Group \\ on Geriatrics, Charité - Universitätsmedizin Berlin, Berlin, Germany, ${ }^{10}$ Department of Nutrition and Gerontology, German Institute of Human Nutrition Potsdam Rehbrücke, \\ Germany
}

\section{Abstract}

Background Body weight loss is a frequent complication after stroke, and its adverse effect on clinical outcome has been shown in several clinical trials. The purpose of this prospective longitudinal single-centre observational study was to investigate dynamical changes of body composition and body weight after ischemic stroke and an association with functional outcome. Methods Sixty-seven consecutive patients (age $69 \pm 11$ years, body mass index $27.0 \pm 4.1 \mathrm{~kg} / \mathrm{m}^{2}$, 42\% female patient, mean \pm SD) with acute ischemic stroke with mild to moderate neurological deficit (National Institute of Health Stroke Scale median 4, ranged 0-12) were analysed in the acute phase (4 \pm 2 days) and at 12 months (389 \pm 26 days) follow-up. Body composition was examined by dual energy X-ray absorptiometry. Cachexia was defined according to the consensus definition by body weight loss $\geq 5 \%$ within 1 year and additional clinical signs. Lean tissue wasting was considered if a ratio of upper and lower limbs lean mass sum to squared height $\left(\mathrm{kg} / \mathrm{m}^{2}\right)$ was $\leq 5.45 \mathrm{~kg} / \mathrm{m}^{2}$ for female patient and $\leq 7.25 \mathrm{~kg} / \mathrm{m}^{2}$ for male patient. Results According to the body weight changes after 12 months, 42 (63\%) patients had weight gain or stable weight, 11 (16\%) patients had moderate weight loss, and 14 (21\%) patients became cachectic. A relative decline of $19 \%$ of fat tissue and $6.5 \%$ of lean tissue was observed in cachectic patients, while no changes of lean tissue were observed in non-cachectic patients after 12 months. The modified Rankin Scale was $48 \%$ higher $(2.1 \pm 1.6, P<0.05)$, Barthel Index was $22 \%$ lower $(71 \pm 39, P<0.01)$, and handgrip strength was $34 \%$ lower $(21.9 \pm 13.0, P<0.05)$ in cachectic compared to non-cachectic patients after 12 months. The low physical performance if defined by Barthel Index $<60$ points was linked to the lean tissue wasting $(O R 44.8, P<0.01)$, presence of cachexia (OR 20.8, $P<0.01$ ), and low body mass index $<25 \mathrm{~kg} / \mathrm{m}^{2}$ (OR $11.5, P<0.05$ ). After adjustment for cofounders, lean tissue wasting remained independently associated with the low physical performance at 12 months follow-up (OR 137.9, $P<0.05$ ).

Conclusions In this cohort study, every fifth patient with ischemic stroke fulfilled the criteria of cachexia within 12 months after index event. The incidence of cachexia was $21 \%$. Cachectic patients showed the lowest functional and physical capacity.

Keywords Body weight; Body composition; DXA; Stroke; Cachexia 


\section{Introduction}

Stroke is a leading medical, socio-economic and health care problem worldwide. Increasing global life expectancy and a reduction of the acute post-stroke mortality rate contribute to the rising costs in stroke care. ${ }^{1-3}$ About two thirds of the patients remain disabled after stroke. ${ }^{4,5}$ The course of poststroke recovery depends on the initial stroke severity and stroke-related complications including inflammation, infection, metabolic dysfunction, and degree of disability. Several clinical trials have shown an important role of body weight and nutritional status at stroke onset for the functional outcome and mortality after stroke ${ }^{6-8}$ and an association between the body weight and mortality in experimental stroke. ${ }^{9}$

Body weight loss after stroke is a common observation in acute and chronic stroke. ${ }^{6}$ However, detailed information on body composition changes after stroke are scarce. ${ }^{10,11}$ Standardized assessment of body composition is feasible using dual-energy X-ray absorptiometry (DXA), bioelectrical impedance, or computer tomography. ${ }^{12-14}$ Tissue wasting or cachexia is a complex metabolic syndrome of multifactorial origin $^{15}$ that has been most frequently shown in association with several chronical diseases including chronic heart failure, chronic obstructive pulmonary disease, kidney disease, or cancer. ${ }^{16-19}$ The reported prevalence of cachexia ranges between $5 \%$ and $80 \%$ depending on disease and disease severity and has been linked to poor outcome. ${ }^{20,21}$ However, the incidence of cachexia has not been studied in detail in patients with ischemic stroke.

The aim of this prospective observational study was to analyse changes of body weight and body composition and to investigate the functional outcome in cachectic and noncachectic patients with stroke.

\section{Methods}

\section{Ethical conduct and study population}

The investigator-initiated single-centre longitudinal prospective observational body size in stroke study ${ }^{22}$ (German registry for clinical trials number DRKS00000514) was approved by the Ethic Committee of Charité-Universitätsmedizin Berlin, Germany (EA2/008/09), and written informed consent was obtained from all patients.

We studied 67 patients with acute ischemic stroke within the territory of the middle cerebral artery. The patients with mild to moderate neurological deficit [defined by the National Institute of Health Stroke Scale (NIHSS) $\leq 12$ points] were consecutively enrolled within $48 \mathrm{~h}$ after stroke onset while being admitted to a stroke unit (Department of Neurology, Charité-Universitätsmedizin Berlin, Campus
Virchow-Klinikum, Berlin, Germany) from June 2009 to November 2012. Following the discharge from the stroke unit, 34 patients were admitted to post-stroke rehabilitation. These patients underwent adjusted early post-stroke rehabilitation programs in specialized rehabilitative clinics according to national standards for rehabilitation procedures after stroke.

Study related examinations included assessments of body composition, physical and functional capacity, muscle strength, and nutritional status and were completed in hospital at baseline ( $4 \pm 2$ days after the index event) and at 12 months follow-up (389 \pm 26 days after stroke onset) visit. According to the changes of the body weight after 12 months, patients were retrospectively grouped into (i) weight gain/stable weight, (ii) moderate weight loss $(<5 \%$ of body weight), and (iii) cachectic subgroups. Cachexia was defined by weight loss $\geq 5 \%$ of the original weight over a period of 12 months and at least three clinical criteria according to the current consensus definition. ${ }^{15}$

\section{Assessment of functional outcome and muscle strength after stroke}

Functional capacity and degree of disability were assessed by the Barthel Index (BI) and by the modified Rankin Scale (mRS). ${ }^{23}$ The BI contains 10 basic activities of the daily living related to self-care and mobility with scores of ' 0 ' to ' 100 ', where the lower scores indicate greater dependency. Low functional status was defined by the $\mathrm{BI}<60$ points.

The mRS measures physical independency by assessment of the body function, activity, and participation in daily tasks on the scale ranging from ' 0 ' (no symptoms) to ' 6 ' (death). Isometric muscle strength of the hand was assessed by the handgrip strength test using a handgrip dynamometer (Saehan Corporation, Korea). The highest of three handgrip measurements of the non-paretic hand was used for analyses.

Maximal isometric muscle strength of the quadriceps muscle (expressed in Newton, $\mathrm{N}$ ) was measured as described previously. ${ }^{24}$ Briefly, the freely hanging legs of the sitting patients were connected at the ankle with a pressure transducer (Multitrace 2, Lectromed, Jersey, Channel Islands), and maximal isometric strength was assessed from the best of three contractions on each leg, with a resting period of at least $60 \mathrm{~s}$ in between.

\section{Body composition}

Body mass index (BMI) was calculated as a ratio of body weight and squared height $\left(\mathrm{kg} / \mathrm{m}^{2}\right)$. For detailed body composition assessment, dual-energy DXA was performed using LunarProdigy densitometer (GE Healthcare, Chalfont St. Giles, UK). Total body scans were analysed to obtain total and regional (upper and lower limbs, and trunk) measurements 
of the fat and lean tissue. The sum of the lean or fat mass of the upper and lower limbs was termed as an appendicular muscle mass (ALM) or appendicular fat mass. The fat-free mass index (FFMI) was calculated as a ratio of ALM and squared height $\left(\mathrm{kg} / \mathrm{m}^{2}\right)$. Lean tissue wasting was defined by low FFMI (for female patient $\leq 5.45 \mathrm{~kg} / \mathrm{m}^{2}$ and for male patient $\leq 7.25 \mathrm{~kg} / \mathrm{m})$.

Appetite was assessed according to the visual analogue scale ranging from ' 0 ' (no appetite) to ' 10 ' (very good appetite). ${ }^{25}$ In addition, nutritional status was assessed by Mini Nutritional Assessment at 12 months as follows: patients were undernourished, if they achieved 16 points or less, at risk for malnutrition if they achieved 17-23.5 points, or had a normal nutritional status if they achieved $\geq 24$ points. $^{26}$

None of the patients included in our study had dysphagia on a clinical relevant level (preventing oral feeding), and none was fed enterally or parenterally.

Blood sampling

Venous blood samples were obtained in all patients after $12 \mathrm{~h}$ of overnight fasting. Standard biochemical parameters were assessed by routine laboratory measurement. Systemic inflammation was present if C-reactive protein (CRP) plasma level was over $6.1 \mathrm{mg} / \mathrm{dL}$, as defined previously. ${ }^{27}$

\section{Statistical analysis}

All data were presented as means \pm standard deviation, median (interquartile range), or percentage as appropriate. All variables were tested for normal distribution using the Kolmogorov-Smirnov test. Non-normally distributed data were log transformed to achieve a normal distribution where indicated. Statistical comparisons were made using paired or unpaired Student's t-tests as appropriate, analysis of variance followed by Fisher's post hoc test, MannWhitney, or Kruskal-Wallis test. Chi-squared test was used to assess categorical distribution between the groups. Pearson's simple regression and logistic regression were used as appropriate. A value of $P<0.05$ was considered statistically significant. Statistical analyses were performed with the StatView 5.0 software package (SAS Institute Inc, Cary, NC) and the software GraphPad Prism 6.0.

\section{Results}

Baseline clinical characteristics of the patients and retrospective study subgroups are presented in Table 1. Twelve months after stroke, 42 (63\%) patients had stable weight or weight gain, $11(16 \%)$ of all patients were found with moderate weight loss, and 14 (21\%) patients became cachectic (Figure 1). There were no significant differences regarding the $\mathrm{BMI}$, the side of stroke-related brain damage, thrombolytic therapy, frequency of paresis, or admission to post-stroke rehabilitation at baseline between all subgroups. Patients who became cachectic 12 months after stroke were significantly older $(P<0.05)$, had more frequently advanced neurological deficit with NIHSS $\geq 5$ (64\%, $P<0.05$ ), had higher degree of dependence by mRS $(P<0.01)$ and $\mathrm{BI}(P<0.05)$, and had the lowest albumin and the highest CRP serum levels $(P<0.001$ and $P<0.01$, respectively) at baseline compared to other subgroups (Table 1).

According to univariate regression analysis, baseline parameters including age, neurological deficit as indicated by a NIHSS $\geq 5$ points, functional dependency as indicated by $\mathrm{mRS}$ and $\mathrm{BI}$, albumin and log-transformed CRP serum levels, systemic inflammation as defined by CRP serum levels $>6.1 \mathrm{mg} / \mathrm{dL}$, self-reported appetite, and handgrip strength were associated with cachexia onset (Table 2). After adjustment for age, sex and BMI, systemic inflammation, and CRP serum levels were independently associated with cachexia development (Table 2).

\section{Body composition}

At baseline, no differences in body composition were observed among study subgroups (Table 3). While in the stable weight/weight gain group no loss of lean mass after 12 months was observed, patients with weight loss showed a reduction in lean mass (Table 3 ). Thus, in patients who became cachectic, a significant reduction of ALM by $6.5 \%(P<0.05)$ was observed (Table 3). The frequency of the lean tissue wasting as defined by FFMI $\leq 5.45 \mathrm{~kg} / \mathrm{m}^{2}$ for female patient and FFMI $\leq 7.25 \mathrm{~kg} / \mathrm{m}^{2}$ for male patient in the cachectic subgroup was 43\% after 12 months $(P<0.001)$ vs. baseline. In addition, appendicular fat mass 12 months after stroke was also the lowest in cachectic patients $(P<0.05)$.

In the stable weight/weight gain group and the moderate weight loss group, no significant reduction of lean tissue according to the FFMI was observed (Table 3). Lean tissue wasting showed no relation to paresis in the studied patients, (OR 1.1, 95\% Cl [0.12-10.6], $P=0.9$ ).

\section{Functional outcome at 12 months follow-up}

Clinical characteristics of the patient subgroups at 12 months follow-up are shown in Table S1. Twelve months after stroke an improvement of functional capacity compared to baseline was found in most patients with stroke (Figure $2 A$ and $2 B$ ). This improvement applied to patients with and without cachexia. Nonetheless, cachectic patients 
Table 1. Clinical characteristics of study cohort at baseline

\begin{tabular}{|c|c|c|c|c|c|}
\hline & Study group & Weight gain/stable weight & Moderate weight loss & Cachexia & \\
\hline Parameter & $n=67$ & $n=42$ & $n=11$ & $n=14$ & $P$-value \\
\hline Age, years & $69 \pm 11$ & $66 \pm 11$ & $70 \pm 10$ & $75 \pm 9$ & 0.03 \\
\hline Male sex; \% (n) & $58(39)$ & $60(25)$ & $82(9)$ & $36(5)$ & 0.07 \\
\hline Body mass index, $\mathrm{kg} / \mathrm{m}^{2}$ & $27.0 \pm 4.1$ & $26.5 \pm 3.7$ & $28.7 \pm 5.1$ & $26.4 \pm 4.8$ & 0.3 \\
\hline Body mass index $<25 \mathrm{~kg} / \mathrm{m}^{2} ; \%(n)$ & $30(20)$ & $31(13)$ & $18(2)$ & $36(5)$ & 0.6 \\
\hline Systolic RR, mmHg & $138 \pm 24$ & $136 \pm 21$ & $150 \pm 27$ & $138 \pm 30$ & 0.2 \\
\hline Diastolic RR, mmHg & $77 \pm 13$ & $77 \pm 12$ & $82 \pm 14$ & $73 \pm 14$ & 0.1 \\
\hline Mean RR, mmHg & $97 \pm 15$ & $96 \pm 14$ & $105 \pm 17$ & $94 \pm 14$ & 0.2 \\
\hline \multicolumn{6}{|l|}{ Stroke severity } \\
\hline Thrombolysis with rt-PA; \% (n) & $31(21)$ & $29(12)$ & $27(3)$ & $43(6)$ & 0.6 \\
\hline Right hemispheric stroke; \% $(n)$ & $66(42)$ & $60(25)$ & $73(8)$ & $75(9)$ & 0.7 \\
\hline Paresis; \% (n) & $84(56)$ & $86(36)$ & $82(9)$ & $79(11)$ & 0.8 \\
\hline Post-stroke rehabilitation; \% (n) & $51(34)$ & $52(22)$ & $46(5)$ & $50(7)$ & 0.9 \\
\hline NIHSS score & $4.5 \pm 3.2$ & $4.3 \pm 3.2$ & $3.5 \pm 2.7$ & $5.8 \pm 3.0$ & 0.2 \\
\hline NIHSS score $5-12 ; \%(n)$ & $37(25)$ & $33(14)$ & $18(2)$ & $64(9)$ & 0.02 \\
\hline Modified Rankin Scale score & $2.0 \pm 1.3$ & $1.8 \pm 1.1$ & $1.6 \pm 1.0$ & $3.0 \pm 1.4$ & 0.003 \\
\hline Modified Rankin Scale score 4-5; \% (n) & $18(12)$ & $12(5)$ & $9(1)$ & $43(6)$ & 0.02 \\
\hline Barthel Index score & $78 \pm 29$ & $82 \pm 26$ & $88 \pm 20$ & $60 \pm 38$ & 0.03 \\
\hline Barthel Index score $<60$ & $27(18)$ & $21(9)$ & $12(2)$ & $50(7)$ & 0.09 \\
\hline \multicolumn{6}{|l|}{ Comorbidities } \\
\hline Diabetes mellitus; \% ( $n$ ) & $27(18)$ & $17(7)$ & $55(6)$ & $36(5)$ & 0.03 \\
\hline Hypertension; \% (n) & $84(56)$ & $83(35)$ & $82(9)$ & $86(12)$ & 0.9 \\
\hline Dyslipidemia; \% (n) & $59(39)$ & $55(23)$ & $82(9)$ & $50(7)$ & 0.2 \\
\hline \multicolumn{6}{|l|}{ Biochemistry } \\
\hline Haemoglobin, g/dL & $14 \pm 2.0$ & $14.0 \pm 1.9$ & $14.5 \pm 1.1$ & $13.6 \pm 2.7$ & 0.5 \\
\hline Albumin, $g / L$ & $36.8 \pm 5.6$ & $37.4 \pm 5.9$ & $39.1 \pm 3.3$ & $32.2 \pm 5.9$ & 0.001 \\
\hline Glucose, $\mathrm{mg} / \mathrm{dL}$ & $115 \pm 44$ & $114 \pm 46$ & $136 \pm 45$ & $100 \pm 24$ & 0.2 \\
\hline $\mathrm{HbA} 1 \mathrm{c}, \%$ & $6.2 \pm 1.4$ & $6.0 \pm 1.2$ & $6.7 \pm 1.5$ & $6.4 \pm 1.8$ & 0.2 \\
\hline Sodium, mmol/L & $140 \pm 4$ & $141 \pm 3$ & $139 \pm 6$ & $141 \pm 4$ & 0.6 \\
\hline Potassium, $\mathrm{mmol} / \mathrm{L}$ & $4.1 \pm 0.4$ & $4.1 \pm 0.3$ & $4.0 \pm 0.5$ & $4.0 \pm 0.5$ & 0.9 \\
\hline Triglyceride, $\mathrm{mg} / \mathrm{dL}$ & $151 \pm 71$ & $154 \pm 75$ & $142 \pm 43$ & $156 \pm 81$ & 0.7 \\
\hline Cholesterol, mg/dL & $196 \pm 44$ & $198 \pm 45$ & $176 \pm 49$ & $203 \pm 38$ & 0.5 \\
\hline Low density lipoprotein, mg/dL & $114 \pm 42$ & $113 \pm 45$ & $114 \pm 37$ & $128 \pm 26$ & 0.3 \\
\hline High density lipoprotein, mg/dL & $51 \pm 15$ & $54 \pm 17$ & $50 \pm 11$ & $46 \pm 14$ & 0.2 \\
\hline Creatinine, $\mathrm{mg} / \mathrm{dL}$ & $1.0 \pm 0.5$ & $1.1 \pm 0.5$ & $1.1 \pm 0.3$ & $0.9 \pm 0.2$ & 0.8 \\
\hline C-reactive protein, $\mathrm{mg} / \mathrm{L}$ & $3.8[1.8-6.3]$ & $3.4[1.7-6.6]$ & $1.7[1.0-4.8]$ & $8.8[4.6-22.4]$ & 0.002 \\
\hline Uric acid, $\mathrm{mg} / \mathrm{dL}$ & $5.7 \pm 1.4$ & $5.6 \pm 1.5$ & $5.7 \pm 1.3$ & $5.9 \pm 1.2$ & 0.9 \\
\hline
\end{tabular}

NIHSS, National Institute of Health Stroke Scale.

remained with the more severe disability as shown by the higher $\mathrm{mRS}$ score $(2.1 \pm 1.6, P<0.01)$ and lower $\mathrm{BI}$ score (71 $\pm 39, P<0.01)$ compared to other study subgroups (Figure $2 A$ and $2 B$ ). Better functional capacity according to the $\mathrm{BI}$ was associated with weight gain $(R=0.25$, $P<0.05)$ in all patients with stroke (Figure $2 C$ ).

Cachectic patients showed the lowest muscle strength as assessed by handgrip strength test and quadriceps strength test (Figure $3 A$ and $3 B$ ). An association between the FFMI and handgrip strength was observed in non-cachectic $(R=0.54, P<0.0001)$ and in cachectic $(R=0.71, P<0.01)$ patients at 12 months follow-up (Figure $3 C$ ).

Univariate logistic regression analysis showed an association of low functional status as defined by $\mathrm{BI}$ score $<60$ points with the lean tissue wasting, presence of cachexia, BMI, body weight loss, nutritional status, low albumin, and high CRP serum levels (Table 4). After adjustment for covariates included age and sex, lean tissue wasting and FFMI remained independently associated with low functional status.

\section{Nutritional status at 12 months follow-up}

Significant improvement of the appetite according to the visual appetite scale was observed in patients with weight gain/stable weight but not in those with any degree of weight loss (Figure 4A). Cachectic patients were found with the lowest appetite and nutritional status (Figure $4 A$ and $4 B)$. A direct association between the weight gain and appetite was observed ( $R=0.4, P<0.002$, Figure $4 C$ ).

\section{Systemic inflammation}

At 12 months follow-up, inflammatory activity was still ongoing in cachectic patients [median CRP 7.6 (interquartile range $2.6-15.2) \mathrm{mg} / \mathrm{dL}, P<0.01$ ] compared to the other subgroups (Table S1). Lean tissue wasting, nutritional status, selfreported appetite, $\mathrm{mRS}, \mathrm{BI}$, and handgrip strength were associated with systemic inflammation (Table 5). 
Figure 1 Body weight changes at 12 months follow-up in the study population. Incidence of cachexia.

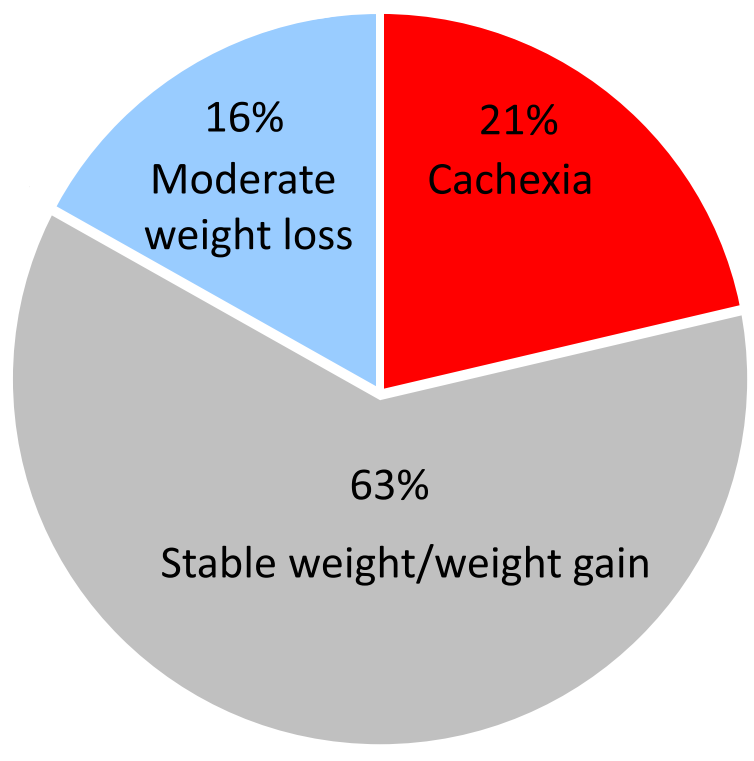

\section{Discussion}

Our study demonstrates an incidence of cachexia of $21 \%$ within 1 year after stroke. Physical and functional clinical status at 12 months follow-up was lower in cachectic patents compared to non-cachectic patients with stroke. We also identified clinical parameters predictive for development of cachexia after stroke.

\section{Tissue wasting}

Using DXA analyses, we showed that patients with cachexia, in contrast to patients without cachexia, lost fat mass and lean tissue. Loss of lean mass in paretic patients with stroke, having sedentary lifestyle, is a common observation. ${ }^{28-30}$ However, we observed a lean tissue depletion in cachectic patients regardless of the paresis. Previously, a stroke-related systemic lean tissue wasting due to catabolic activation was reported in a mouse model of middle cerebral artery occlusion. ${ }^{9}$ The extent of the brain damage in mice correlated with apoptotic and proteolytic activity in skeletal muscle in both legs. ${ }^{9}$ In line with this experimental data, we observed an association between the stroke severity and the presence of cachexia.

Certainly, lean tissue decline is an age-dependent phenomenon, with the prevalence ranging between $8 \%$ and $60 \%$ in healthy elders. ${ }^{31-35}$ Previous study showed a prevalence of sarcopenia of $7 \%$ in chronic stroke survivors within 3 years after ischemic or haemorrhagic stroke. ${ }^{10}$ In the present cohort, the prevalence of the lean tissue depletion at the time of the index stroke was within the generally observed age-dependent range. However, an increase from $6 \%$ to $13 \%$ in the prevalence of lean tissue depletion within 12 months with the highest proportion among cachectic patients (43\%) might be linked to stroke-related metabolic imbalance. ${ }^{36}$

\section{Systemic inflammation}

An association between cachexia and inflammatory state in patients with chronic heart failure or chronic obstructive

Table 2. Baseline parameters associated with cachexia onset after stroke

\begin{tabular}{|c|c|c|c|}
\hline Parameter & OR & $95 \% \mathrm{Cl}$ & $P$-value \\
\hline $\begin{array}{l}\text { Presence of } \\
\text { systemic inflammation }\end{array}$ & 8.54 & [2.27-32.17] & 0.002 \\
\hline C-reactive protein, $\log [\mathrm{mg} / \mathrm{L}]$ & 7.93 & {$[1.99-31.60]$} & 0.003 \\
\hline NIHSS 5-12 points & 4.16 & {$[1.20-14.39]$} & 0.02 \\
\hline Barthel Index $<60$ points & 3.80 & {$[1.10-13.20]$} & 0.03 \\
\hline Modified Rankin Scale, point & 2.19 & {$[1.32-3.65]$} & 0.003 \\
\hline Age, year & 1.08 & {$[1.01-1.15]$} & 0.02 \\
\hline Barthel Index, 10 points & 0.78 & {$[0.64-0.95]$} & 0.02 \\
\hline Handgrip strength, kg & 0.91 & {$[0.85-0.98]$} & $<0.01$ \\
\hline Albumin, $\mathrm{g} / \mathrm{dL}$ & 0.79 & {$[0.68-0.92]$} & 0.002 \\
\hline Appetite, point VAS & 0.51 & {$[0.34-0.77]$} & 0.002 \\
\hline Appendicular lean mass, kg & 0.83 & [0.70-0.99] & 0.03 \\
\hline Lean mass upper limbs, kg & 0.58 & {$[0.34-0.99]$} & $<0.05$ \\
\hline Lean mass lower limbs, kg & 0.76 & {$[0.60-0.96]$} & 0.02 \\
\hline \multicolumn{4}{|l|}{$\begin{array}{l}\text { Multivariable logistic model } \\
\text { adjusted for age, sex, and BMI }\end{array}$} \\
\hline I. Systemic inflammation & 9.66 & {$[1.96-47.63]$} & 0.005 \\
\hline II. C-reactive protein, log[mg/L] & 6.67 & {$[1.34-33.12]$} & 0.02 \\
\hline
\end{tabular}

NIHSS, National Institute of Health Stroke Scale; VAS, visual analogue scale. 
Table 3. Body composition in patient subgroups

\begin{tabular}{|c|c|c|c|c|c|c|}
\hline \multirow[b]{2}{*}{ Parameter } & \multicolumn{2}{|c|}{ Weight gain/stable weight } & \multicolumn{2}{|c|}{ Moderate weight loss } & \multicolumn{2}{|l|}{ Cachexia } \\
\hline & Baseline & 12 months & Baseline & 12 months & Baseline & 12 months \\
\hline Body mass index, $\mathrm{kg} / \mathrm{m}^{2}$ & $26.7 \pm 3.5$ & $28.2 \pm 4.9^{* * *}$ & $28.7 \pm 5.1$ & $27.9 \pm 4.9$ ** & $26.4 \pm 4.8$ & $3 \quad 24.2 \pm 4.5^{* * \#++\dagger}$ \\
\hline FFMI, $\mathrm{kg} / \mathrm{m}^{2}$, female patient & $6.4 \pm 0.8$ & $6.6 \pm 0.7$ & $6.5 \pm 0.04$ & $6.6 \pm 0.1$ & $6.1 \pm 0.8$ & $6.0 \pm 1.3^{\dagger}$ \\
\hline $\begin{array}{l}\mathrm{FFMI}, \mathrm{kg} / \mathrm{m}^{2} \text {, male patient } \\
\text { Lean mass, } \mathrm{kg}\end{array}$ & $8.3 \pm 0.7$ & $8.3 \pm 0.8$ & $8.1 \pm 0.7$ & $7.9 \pm 0.7$ & $7.6 \pm 0.5$ & $7.4 \pm 0.6^{* \#}$ \\
\hline $\begin{array}{l}\text { Lean mass, } \mathrm{kg} \\
\text { Appendicular }\end{array}$ & $22.2 \pm 4.9$ & $22.4 \pm 4.7$ & $22.7 \pm 3.9$ & $22.3 \pm 3.5$ & $18.6 \pm 4.5$ & $18.0 \pm 4.6^{* \#+\dagger}$ \\
\hline Lower limbs & $16.9 \pm 3.4$ & $17.2 \pm 3.3^{*}$ & $17.7 \pm 3.5$ & $17.7 \pm 3.0$ & $14.2 \pm 3.2$ & $13.9 \pm 3.4^{\dagger \dagger}$ \\
\hline Upper limbs & $5.4 \pm 1.6$ & $5.3 \pm 1.5$ & $5.8 \pm 1.1$ & $5.1 \pm 1.4^{* *}$ & $4.4 \pm 1.3$ & $4.1 \pm 1.3^{* * \#+}$ \\
\hline Fat mass, kg & & & & & & \\
\hline Appendicular & $11.9 \pm 3.8$ & $12.1 \pm 3.9 * *$ & $10.2 \pm 3.2$ & $9.2 \pm 3.2^{* \#}$ & $10.0 \pm 4.1$ & $9.1 \pm 4.4^{* * \#}$ \\
\hline Lower limbs & $9.1 \pm 2.9$ & $9.8 \pm 3.4$ & $8.7 \pm 2.7$ & $7.6 \pm 2.8^{* \dagger}$ & $7.8 \pm 2.9$ & $7.6 \pm 3.6^{* *}$ \\
\hline Upper limbs & $2.4 \pm 0.8$ & $2.5 \pm 0.8$ & $2.4 \pm 0.8$ & $1.9 \pm 0.5^{*}$ & $2.3 \pm 1.0$ & $2.0 \pm 1.3^{* * \dagger}$ \\
\hline
\end{tabular}

FFMI, fat-free mass index.

${ }^{*} P<0.05$.

$* * P<0.01$

$* * * P<0.001$ vs. baseline.

${ }^{\#} P<0.05$.

${ }^{\dagger} P<0.01$ vs. weight gain/stable weight group.

${ }^{\mathrm{tt}} P<0.05$

${ }^{\dagger+\dagger} P<0.001$ vs. moderate weight loss group.

Figure 2 (A) Assessment of functional capacity according to the Barthel Index in study subgroups. (B) Assessment of degree of disability according to the modified Rankin Scale in study subgroups. (C) Relationship between the functional outcome and weight change at 12 months follow-up

A
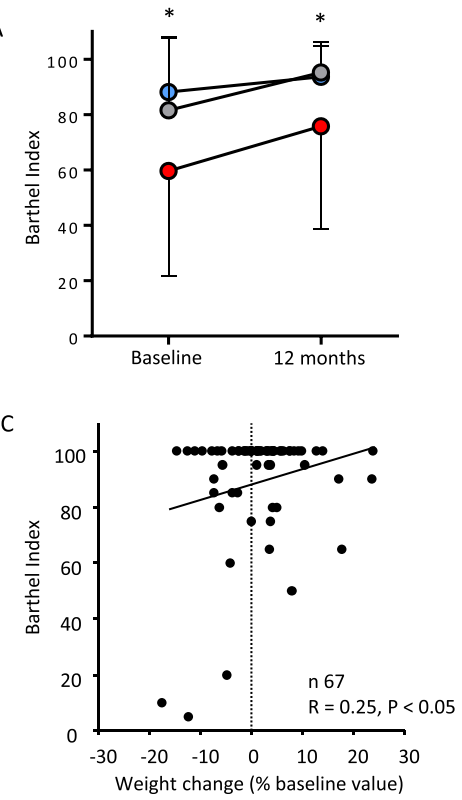

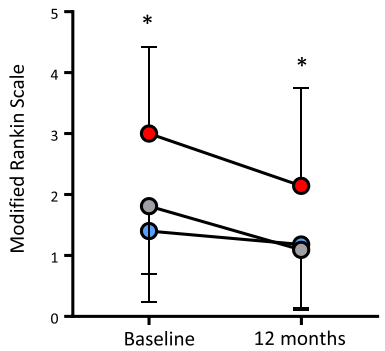

pulmonary disease has been shown previously. ${ }^{25,37}$ In the present study, cachectic patients showed similar signs of inflammatory activation with elevated CRP levels assessed at 12 months follow-up accompanied by lean tissue depletion, low functional capacity, and reduction of body weight. A relation between systemic inflammation and loss of muscle strength and muscle thickness in population-based clinical trials has been reported previously. ${ }^{27,38}$ In experimental setting, administration of the inflammatory markers in rats caused muscle protein breakdown. ${ }^{39}$ Thus, our results were in accordance with previous observations.

Our recent studies investigating cachexia in patients with chronic heart failure suggested an association of cardiac cachexia with gastrointestinal congestion, increased concentration of gut bacteria, and systemic inflammation. ${ }^{37,40}$ Further, changes in gut microbiota after stroke were linked to increased pro-inflammatory cytokines levels. ${ }^{41}$ Several pathways linking systemic inflammation to the brain and 
Figure 3 (A) Maximal handgrip strength in study subgroups. (B) Maximal quadriceps strength in study subgroups. (C) Association of fat-free mass index with maximal handgrip strength in patients with and without cachexia at 12 months follow-up

A

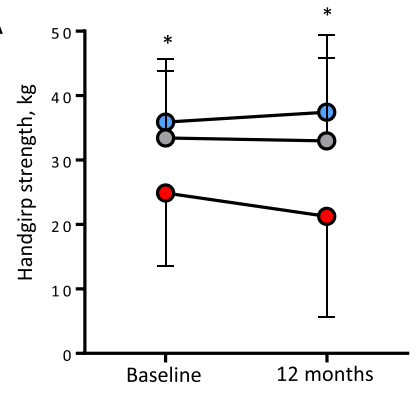

C

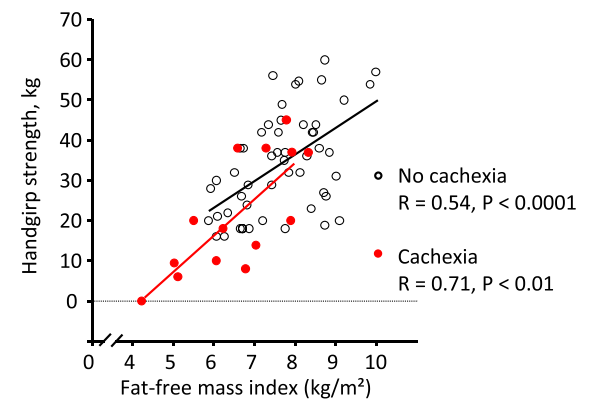

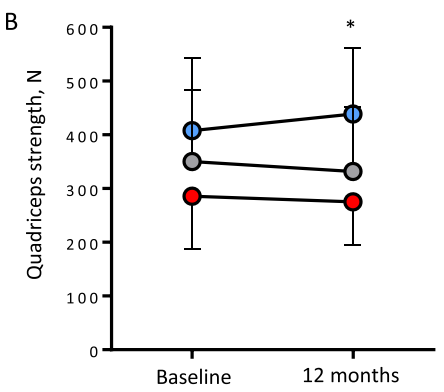

Table 4. Risk factors associated with functional dependency defined by Barthel Index $<60$ at 12 months follow-up

\begin{tabular}{|c|c|c|c|}
\hline Parameter & OR & $95 \% \mathrm{Cl}$ & $P$-value \\
\hline Lean tissue wasting & 44.8 & [4.17-481.56] & 0.002 \\
\hline Presence of cachexia & 20.8 & [2.09-206.15] & $<0.01$ \\
\hline Undernourished/at risk for malnutrition & 14.7 & [0.99-215.37] & 0.05 \\
\hline Body mass index $<25 \mathrm{~kg} / \mathrm{m}^{2}$ & 11.5 & [1.19-110.67] & 0.04 \\
\hline C-reactive protein, $\log [\mathrm{mg} / \mathrm{L}]$ & 10.1 & {$[1.39-73.54]$} & 0.02 \\
\hline NIHSS, point & 1.43 & {$[1.07-1.90]$} & 0.02 \\
\hline Delta weight loss, kg & 0.82 & [0.68-0.99] & 0.04 \\
\hline Body mass index, $\mathrm{kg} / \mathrm{m}^{2}$ & 0.68 & {$[0.50-0.93]$} & 0.02 \\
\hline MNA scale, point & 0.57 & {$[0.37-0.87]$} & 0.01 \\
\hline Albumin, $\mathrm{g} / \mathrm{L}$ & 0.75 & {$[0.60-0.94]$} & 0.01 \\
\hline $\mathrm{FFMI}, \mathrm{kg} / \mathrm{m}^{2}$ & 0.16 & {$[0.04-0.63]$} & 0.01 \\
\hline Lean mass arms, $\mathrm{kg}$ & 0.24 & {$[0.06-0.92]$} & 0.04 \\
\hline Lean mass legs, kg & 0.58 & [0.38-0.89] & 0.01 \\
\hline Appendicular lean mass, kg & 0.66 & {$[0.47-0.93]$} & 0.02 \\
\hline Handgrip strength, $\mathrm{kg}$ & 0.89 & {$[0.81-0.99]$} & 0.04 \\
\hline \multicolumn{4}{|c|}{ Multivariable logistic model adjusted for age and sex } \\
\hline I. Lean tissue wasting & 137.9 & {$[2.04-9324.7]$} & 0.02 \\
\hline II. FFMI, $\mathrm{kg} / \mathrm{m}^{2}$ & 0.11 & {$[0.13-0.99]$} & $<0.05$ \\
\hline
\end{tabular}

FFMI, fat-free mass index; NIHSS, National Institute of Health Stroke Scale; MNA, Mini Nutritional Assessment.

responsible for development of 'sickness behaviour' with attenuated parasympathetic tone, reduced appetite, altered thermoregulation, and impaired energy metabolism were previously described. ${ }^{42}$ Accordingly, we observed an association between systemic inflammation and reduced nutritional status in patients with stroke. Cachectic patients had the lowest appetite leading to decreased food intake. An involvement of anorexia in regulation of body composition in chronic inflammatory disease and cachexia development was suggested. ${ }^{25}$ Therefore, ongoing systemic inflammation in chronic stroke might lead to increased metabolic drive, appetite loss, and energetic deficit resulting in proteolytic breakdown and tissue wasting. ${ }^{25,43}$

\section{Functional limitations}

In the present study, we showed implications of cachexia on functional outcome after stroke. Cachectic patients had the 
Figure 4 (A) Self-reported appetite score according to visual analogue scale in study subgroups. (B) Nutritional status according to Mini Nutritional Assessment score in patient subgroups at 12 months follow-up. (C) Association of body weight change with score by visual analogue scale at 12 months follow-up

A

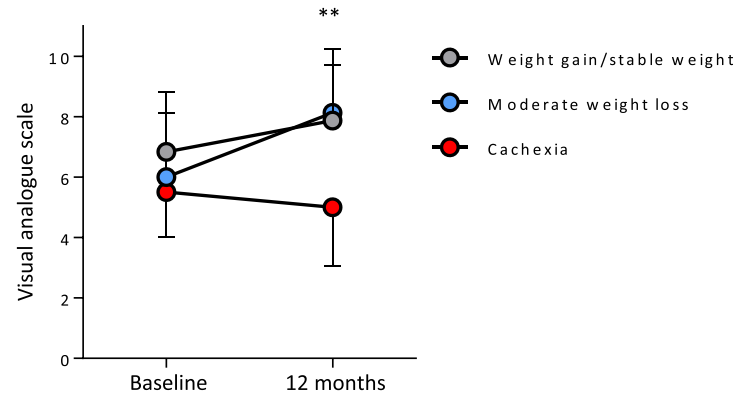

C

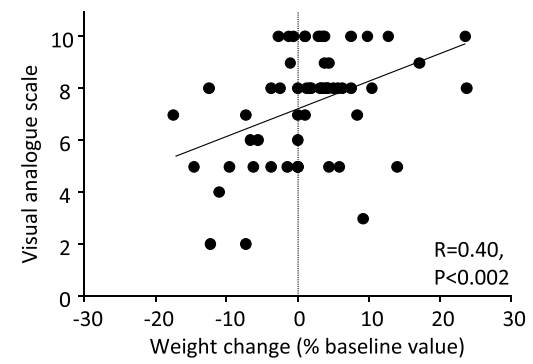

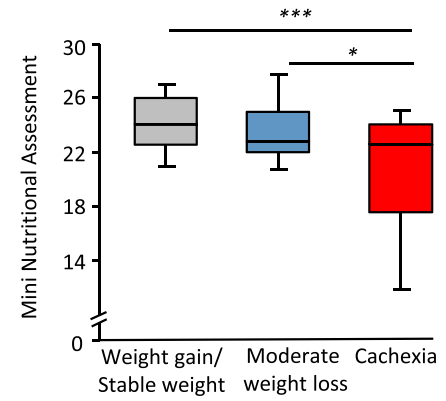

Table 5. Risk factors associated with the presence of systemic inflammation at 12 months follow-up serving as dependent variable

\begin{tabular}{lccr}
\hline Parameter & OR & $95 \% \mathrm{Cl}$ & $P$-value \\
\hline Lean tissue wasting & 5.20 & {$[0.99-27.09]$} & 0.05 \\
Changes of body weight, kg & 0.89 & {$[0.80-1.01]$} & 0.06 \\
Appetite according to VAS, point & 0.71 & {$[0.52-0.97]$} & 0.03 \\
Undernourished/at risk for malnutrition & 0.81 & {$[0.69-0.99]$} & 0.04 \\
Modified Rankin Scale, point & 1.96 & {$[1.16-3.31]$} & 0.01 \\
Barthel Index, 10 points & 0.68 & {$[0.49-0.95]$} & 0.02 \\
Handgrip strength, kg & 0.94 & {$[0.89-0.99]$} & 0.02 \\
\hline
\end{tabular}

VAS, visual analogue scale.

most severe stroke-related disability across the study groups as assessed by the $\mathrm{BI}$ and $\mathrm{mRS}$, although they significantly improved their functional status at 12 months follow-up. We showed that the presence of cachexia and the lean tissue depletion were associated with the low functional capacity as defined by the $\mathrm{BI} .^{23}$ Further, both lower BMI and higher body weight loss were more frequently observed in patients with higher degree of functional dependence. Therefore, our findings are in accordance with the previous clinical trials, investigating cachexia in chronic diseases and suggesting its unfavourable impact on activities of daily living, clinical and functional outcome. ${ }^{25,37}$

Additionally, to the $\mathrm{BI}$ and $\mathrm{mRS}$, the study protocol considered a performance of the short physical performance battery (SPPB). ${ }^{22}$ SPPB is widely used in geriatric medicine and includes examination of standing ability, time walking of 3 or $4 \mathrm{~m}$, and time to rise from the chair. ${ }^{44}$ It became apparent in the study that the SPPB was less suitable to evaluate the functional capacity due to coordination deficit if patient had a paretic limb. Hence, merely $51 \%$ of all patients (i.e. $21 \%$ of the cachectic group) at baseline and $82 \%$ of the patients (57\% of the cachectic group) at 12 months follow-up were able to complete the SPPB, which indicates a relevant floor effect as discussed previously. ${ }^{12}$ We performed a $4 \mathrm{~m}$ of gait test as a part of the SPPB during the baseline and 12 months follow-up. The gait speed at baseline was $1.1 \pm 0.3$ vs. $0.9 \pm 0.2$ vs. $0.8 \pm 0.4 \mathrm{~m} / \mathrm{s}, P=0.3$ in the stable weight vs. moderate weight loss vs. cachectic subgroup, respectively. Twelve months after stroke, gait speed remained identical in all three groups due to the high frequency of paresis in these patients. Thus, due to the relevant floor effect of the test battery in the setting of stroke as well as limited insight into the functional capacity of the patients, we decided to omit these data. 


\section{Weight gain}

The majority of patients in the present study increased the weight and improved their functional capacity during the first year after stroke. We observed a correlation between increasing weight and better functional outcome with higher $\mathrm{BI}$, indicating a positive effect of 'obesity paradox'. ${ }^{6}$

\section{Limitations}

Our study has several limitations. First, the number of patients with stroke is limited. Second, only study patients with mild to moderate stroke deficit were included, thus our results are not generalizable to patients with severe stroke. Therefore, further longitudinal studies are warranted to confirm and extend our findings.

\section{Conclusions}

The observed incidence of cachexia was $21 \%$ among the patients with stroke at 12 months follow-up. Overall, more fragile patients defined by higher age, advanced neurological deficit, and functional disability were at risk for cachexia. At 12 months follow-up, cachectic patients remained with the lowest functional and physical status. Development of cachexia after stroke should be recognized as a relevant complication. Better understanding of the interaction between stroke-related brain injury and systemic metabolism seems to be required for better prevention of cachexia in patients with stroke.

\section{Acknowledgements}

The authors certify that they comply with the ethical guidelines for publishing in the Journal of Cachexia, Sarcopenia and Muscle: update $2017 .^{45}$

\section{Funding}

The Center for Stroke Research Berlin (CSB) is an integrated research and treatment centre. The body size in stroke study received funding from German Federal Ministry of Education and Research (BMBF). We acknowledge support from the German Research Foundation (DFG) and the Open Access Publication Fund of Charité - Universitätsmedizin Berlin.

\section{Online supplementary material}

Additional supporting information may be found online in the Supporting Information section at the end of the article.

Table S1: Clinical characteristics of patient subgroups at 12 months follow-up

\section{Conflict of Interest}

None declared.

\section{References}

1. Gaeta M, Campanella F, Gentile L, Schifino GM, Capasso L, Bandera F, et al. European cardiovascular mortality over the last three decades: evaluation of time trends, forecasts for 2016. Ann Ig 2017;29:206-217.

2. Benjamin EJ, Virani SS, Callaway CW, Chang AR, Cheng S, Chiuve SE, et al. Heart disease and stroke statistics-2018 update: a report from the American Heart Association. Circulation 2018;137:1-442.

3. Feigin VL, Norrving B, Mensah GA. Global burden of stroke. Circ Res 2017;120:439-448.

4. Sousa RM, Ferri CP, Acosta D, Albanese E, Guerra M, Huang $Y$, et al. Contribution of chronic diseases to disability in elderly people in countries with low and middle incomes: a 10/66 Dementia Research Group populationbased survey. Lancet 2009;374:1821-1830.

5. Feigin VL, Barker-Collo S, Parag V, Senior H, Lawes CM, Ratnasabapathy $Y$, et al. ASTRO study group Auckland stroke outcomes study. Part 1: Gender, stroke types, ethnicity, and functional outcomes 5 years poststroke. Neurology 2010;75:1597-1607.
6. Scherbakov N, Dirnagl U, Doehner W. Body weight after stroke: lessons from the obesity paradox. Stroke 2011;42:3646-3650.

7. Vemmos K, Ntaios G, Spengos K, Savvari P, Vemmou A, Pappa T, et al. Association between obesity and mortality after acute first-ever stroke: the obesity-stroke paradox. Stroke 2011:42:30-36.

8. Doehner W, Schenkel J, Anker SD, Springer J, Audebert HJ. Overweight and obesity are associated with improved survival, functional outcome, and stroke recurrence af ter acute stroke or transient ischaemic attack: observations from the TEMPiS trial. Eur Heart J 2013;34:268-277.

9. Springer J, Schust S, Peske K, Tschirner A, Rex A, Engel $O$, et al. Catabolic signaling and muscle wasting after acute ischemic stroke in mice: indication for a stroke-specific sarcopenia. Stroke 2014;45:3675-3683.

10. Vahlberg $B$, Zetterberg $L$, Lindmark B, Hellström K, Cederholm T. Functional performance, nutritional status, and body composition in ambulant community-dwelling individuals 1-3 years after suffering from a cerebral infarction or intracerebral bleeding. BMC Geriatr 2016;16:48.

11. Ha L, Hauge T, Iversen PO. Body composition in older acute stroke patients after treatment with individualized, nutritional supplementation while in hospital. BMC Geriatr 2010;10:75.

12. Scherbakov N, Sandek A, Doehner W. Strokerelated sarcopenia: specific characteristics. J Am Med Dir Assoc 2015;16:272-276.

13. Thibault R, Genton L, Pichard C. Body composition: why, when and for who? Clin Nutr 2012;31:435-447.

14. Gonzalez MC, Heymsfield SB. Bioelectrical impedance analysis for diagnosing sarcopenia and cachexia: what are we really estimating? J Cachexia Sarcopenia Muscle 2017;8:187-189.

15. Evans WJ, Morley JE, Argilés J, Bales C, Baracos V, Guttridge D, et al. Cachexia: a new definition. Clin Nutr 2008;27:793-799.

16. Springer J, Springer JI, Anker SD. Muscle wasting and sarcopenia in heart failure 
and beyond: update 2017. ESC Heart Fail 2017;4:492-498.

17. Sanders KJ, Kneppers AE, van de Bool C, Langen RC, Schols AM. Cachexia in chronic obstructive pulmonary disease: new insights and therapeutic perspective. I Cachexia Sarcopenia Muscle 2016;7:5-22.

18. Blauwhoff-Buskermolen S, Langius JAE, Becker A, Verheul HMW. de van der Schueren MAE. The influence of different muscle mass measurements on the diagnosis of cancer cachexia. I Cachexia Sarcopenia Muscle 2017;8:615-622.

19. von Haehling S. Casting the net broader to confirm our imaginations: the long road to treating wasting disorders. I Cachexia Sarcopenia Muscle 2017;8:870-880.

20. Lok C. Cachexia: The last illness. Nature 2015;528:182-183.

21. von Haehling $S$, Anker MS, Anker SD. Prevalence and clinical impact of cachexia in chronic illness in Europe, USA, and Japan: facts and numbers update 2016. J Cachexia Sarcopenia Muscle 2016;7:507-509.

22. Knops $M$, Werner CG, Scherbakov $N$, Fiebach J, Dreier JP, Meisel A, et al. Investigation of changes in body composition, metabolic profile and skeletal muscle functional capacity in ischemic stroke patients: the rationale and design of the body size in stroke study (BoSSS). J Cachexia Sarcopenia Muscle 2013:4:199-207.

23. Kasner SE. Clinical interpretation and use of stroke scales. Lancet Neurol 2006;5:603-612.

24. Doehner W, Turhan G, Leyva F, Rauchhaus $M$, Sandek A, Jankowska EA, et al. Skeletal muscle weakness is related to insulin resistance in patients with chronic heart failure. ESC Heart Fail 2015;2:85-89.

25. Koehler F, Doehner W, Hoernig S, Witt C, Anker SD, John M. Anorexia in chronic obstructive pulmonary disease: association to cachexia and hormonal derangement. Int J Cardiol 2007;119:83-89.

26. Vellas B, Guigoz Y, Garry PJ, Nourhashemi $F$, Bennahum $D$, Lauque $S$, et al. The Min Nutritional Assessment (MNA) and its use in grading the nutritional state of elderly patients. Nutrition 1999;15:116-122.

27. Schaap LA, Pluijm SM, Deeg DJ, Visser M Inflammatory markers and loss of muscle mass (sarcopenia) and strength. Am J Med 2006;119:526, e9-17.

28. English C, McLennan H, Thoirs K, Coates A, Bernhardt J. Loss of skeletal muscle mass after stroke: a systematic review. Int J Stroke 2010;5:395-402.

29. Ryan AS, Dobrovolny CL, Smith GV, Silver $\mathrm{KH}$, Macko RF. Hemiparetic muscle atrophy and increased intramuscular fat in stroke patients. Arch Phys Med Rehabil 2002;83. 1703-1707.

30. Ferrando AA, Paddon-Jones D, Wolfe RR. Bed rest and myopathies. Curr Opin Clin Nutr Metab Care 2006;9:410-415.

31. Legrand D, Vaes B, Matheï C, Swine C, Degryse JM. The prevalence of sarcopenia in very old individuals according to the European consensus definition: insights from the BELFRAIL study. Age Aging 2013;42:727-734.

32. Abellan van Kan G. Epidemiology and consequences of sarcopenia. J Nutr Health Aging 2009;13:708-712.

33. Baumgartner RN, Koehler KM, Gallagher D, Romero L, Heymsfield SB, Ross RR, et al. Epidemiology of sarcopenia among the elderly in New Mexico. Am J Epidemiol 1998;147:755-763.

34. Janssen I, Heymsfield SB, Ross R. Low relative skeletal muscle mass (sarcopenia) in older persons is associated with functional impairment and physical disability. J Am Geriatr Soc 2002;50:889-896.

35. Cherin $\mathrm{P}$, Voronska $\mathrm{E}$, Fraoucene $\mathrm{N}$, de Jaeger C. Prevalence of sarcopenia among healthy ambulatory subjects: the sarcopenia begins from 45 years. Aging Clin Exp Res 2014;26: 137-146.

36. Scherbakov N, von Haehling S, Anker SD, Dirnagl U, Doehner W. Stroke induced Sarcopenia: muscle wasting and disability after stroke. Int J Cardiol 2013;170:89-94.
37. Sandek A, Swidsinski A, Schroedl W, Watson A, Valentova M, Herrmann R, et al. Intestinal blood flow in patients with chronic heart failure: a link with bacterial growth, gastrointestinal symptoms, and cachexia. J Am Coll Cardiol 2014;64:1092-1102.

38. Nozoe $M$, Kanai $M$, Kubo $H$, Kitamura $Y$, Yamamoto M, Furuichi A, et al. Shimada $S$ changes in quadriceps muscle thickness, disease severity, nutritional status, and creactive protein after acute stroke. J Stroke Cerebrovasc Dis 2016;25:2470-2474.

39. Goodman MN. Tumor necrosis factor induces skeletal muscle protein breakdown in rats. Am J Physiol 1991;260:E727-E730.

40. Valentova $M$, von Haehling $S$, Bauditz $J$, Doehner W, Ebner N, Bekfani T, et al. Intestinal congestion and right ventricular dysfunction: a link with appetite loss, inflammation, and cachexia in chronic heart failure. Eur Heart J 2016;37:1684-1691.

41. Wen SW, Wong CHY. An unexplored braingut microbiota axis in stroke. Gut Microbes 2017;8:601-606.

42. Dantzer R, O'Connor JC, Freund GG, Johnson RW, Kelley KW. From inflammation to sickness and depression: when the immune system subjugates the brain. Nat Rev Neurosci 2008;9:46-56.

43. Braun TP, Marks DL. Pathophysiology and treatment of inflammatory anorexia in chronic disease. J Cachexia Sarcopenia Muscle 2010;1:135-145.

44. Guralnik JM, Simonsick EM, Ferrucci L, Glynn RJ, Berkman LF, Blazer DG, et al. Wallace RB. A short physical performance battery assessing lower extremity function: association with self-reported disability and prediction of mortality and nursing home admission. J Gerontol 1994;49: M85-M94.

45. von Haehling S, Morley JE, Coats AJS, Anker SD. Ethical guidelines for publishing in the Journal of Cachexia, Sarcopenia and Muscle: update 2017. J Cachexia Sarcopenia Muscle 2017;8:1081-1083. 